\title{
L'HUMANISME RATIONNEL DE FW (FRANCIS WOLFF)
}

\author{
Frédéric WORMS
}

ENS Paris

Sous le nom d'«humanisme rationnel de FW », - c'est-à-dire ici bien sûr de Francis Wolff - nous n'entendons pas caractériser seulement la personnalité d'un homme, d'un philosophe et d'un ami, mais bel et bien définir une doctrine, et même une doctrine complète et centrale de notre temps, non seulement pour lui rendre l'hommage qu'elle exige, mais aussi pour reprendre la conversation avec elle et avec lui.

Cette doctrine, que nous caractériserons donc comme humanisme rationnel, répond d'une manière nouvelle et originale, mais encore une fois complète et centrale, aux exigences traditionnelles de l'humanisme qui sont elles-mêmes plus complexes et plus exigeantes que l'on ne croit d'habitude. Et c'est ce qu'il faut rappeler d'abord avant de lui adresser la question qui nous semble néanmoins plus secrètement encore cachée en son cœur, comme elle l'est aussi dans notre temps.

Mais quelles sont donc, tout d'abord, les exigences réelles d'un humanisme digne de ce nom?

Nous en verrions trois. Pour parler d'humanisme, il faut d'abord, bien entendu, revendiquer une spécificité irréductible et caractéristique de l'homme ou de l'humain, parmi les êtres ou du moins, si l'on veut, parmi les vivants. Ce n'est déjà pas si simple, et cela a souvent été contesté. On l'a souvent cherchée dans la «raison», mais si Francis Wolff revient à ce geste, ce n'est pas au sens traditionnel de ce geste. Selon quelle méthode cherche-t-il à démontrer une spécificité rationnelle de «l'homme » ? On est curieux et on sera surpris de le savoir. Mais, pour parler d'humanisme, constater une spécificité de l'homme ou de l'être humain ne suffit pas. Encore faut-il y trouver une « valeur ». Là aussi, il y eut de nombreuses versions de cette « valeur » de l'humain : ou bien valeur « en soi » dans l'être, qu'il faut donc conduire tous les êtres à respecter ; ou bien «valeur » a minima, pour l'être humain lui-même, qui devrait donc orienter et normer son action sur ce qui le définit lui-même dans son être. Rien d'évident, d'aucun de ces deux côtés : comment prouver cette valeur de l'humain par rapport aux autres êtres et même comme principe de l'action des êtres humains? Et cela ne suffit pas 
encore. Parler d'humanisme, c'est soutenir que tous les êtres humains ont accès à cet être et à cette valeur de l'homme, et inversement (ce qui est non moins important), c'est trouver ce principe de l'humain à l'œuvre dans tout ce que font les humains. Rien d'humain ne doit être «étranger» à l'humaniste, qui œuvre aussi à ce que la connaissance de l'humain soit accessible à tous les humains, qui la portent en eux le plus souvent sans le savoir. Enlevez une de ces dimensions à l'humanisme et vous n'en aurez qu'une caricature, qui l'affaiblit en son principe et face à ses adversaires, qui ne sont pas toujours des antihumanistes seulement en théorie, mais qui le sont souvent aussi en pratique. Inversement, on peut discuter une grande doctrine humaniste en contestant un point constitutif de l'humanisme et en se déclarant anti-humaniste, mais on peut aussi le faire au nom de l'humanisme lui-même et en approfondissant une des dimensions constitutives pour le renforcer encore en en partageant le souci et le problème. Telle sera, on le devine, notre direction et notre question, dès que l'on aura parcouru rapidement ce qui constitue l'humanisme rationnel de FW, sa doctrine qui rejoint si profondément aussi sa personne, comme il se doit en général et peut-être plus encore pour un humanisme philosophique.

Mais une fois caractérisées rapidement les exigences de l'humanisme en général, examinons à présent les caractéristiques de l'humanisme de Francis Wolff.

Elles répondent à ces exigences générales, d'une manière qui se veut démonstrative et donc bien sûr rationnelle, mais qui passe à chaque fois par un détour que l'on peut dire empirique, ou factuel, et donc aussi par une épreuve et par une surprise, qui font l'originalité et la force de cette doctrine nouvelle, pour notre temps. On peut nommer rapidement ces trois détours ou ces trois surprises: il s'agit de l'histoire, du langage, et de ce que nous appellerons (comment faire autrement) la vie.

Il y a bien sûr l'histoire, tout d'abord et en premier lieu, et pas seulement parce que notre FW est d'abord un historien, en l'occurrence de la philosophie antique, mais bien plus largement, et jusqu'à une histoire d'un présent qui ne se définit comme tel, comme il se doit, que dans et grâce à une histoire. Mais il s'agit bien d'abord de la philosophie antique. Or, que constate ici Francis Wolff, dans toute la partie de son œuvre qui y est consacrée et qui culmine sans doute dans le recueil significativement intitulé L'être, l'homme, le disciple ? Ce qu'il constate, c'est que la figure grecque de l'homme comme « animal rationnel» n'est que la première figure de la rationalité de l'homme, qu'elle a des caractéristiques précises, datées, historiques donc, mais qu'elle ouvre aussi sur le problème transhistorique et même dit-il aussi «anhistorique » (introduction, p. 10) de la figure de l'homme rationnel. Il y a une «configuration» 
grecque qui situe la question de l'homme, au carrefour des mathématiques et de la métaphysique notamment, comme inscrit dans une "raison » qui organise l'être et le monde. Mais ce qui demeure et de manière transhistorique, c'est bien plutôt (et on sent là une relecture d'une originalité frappante de l'un des maîtres de FW, à savoir Michel Foucault), la figure de l'homme et de la raison, au carrefour des savoirs, bien plutôt qu'au principe des savoirs, encore moins, comme à l'âge moderne, au fondement de tous les savoirs, cette figure fondatrice de l'homme rationnel n'étant qu'une transformation historique de plus de ce problème transhistorique de l'humain au carrefour de tous les savoirs rationnels. Le moment grec incarné alors de manière emblématique par Socrate est celui de l'ouverture de cette question, qui sera remise en question, dès que l'un de ses piliers, qu'il s'agisse d'un savoir fondamental ou de l'autre, sera lui-même bouleversé dans son histoire. Et l'on sait, bien sûr, que FW nous conduit d'Aristote aux neurosciences, dans son enquête historique sur la figure de l'homme au carrefour de toutes les raisons. La méthode est étonnante donc, et relève d'un premier constat qu'elle cherche à imposer : aucune histoire ne peut se passer de ce point géométrique sans cesse rejoué qui est celui de la rationalité humaine. Ainsi, il y a une spécificité de l'humain, comme question qui traverse les temps.

Mais un deuxième chemin s'ouvre alors qui semble contredire le premier, et même contredire la description de cette œuvre comme « humanisme rationnel » : c'est, cette fois, une métaphysique fondée sur une interprétation analytique et anhistorique du langage. C'est la pensée de Dire le monde. Pourquoi et comment faut-il articuler une histoire des figures de l'homme rationnel et une métaphysique analytique du langage? Le point commun est simple pourtant: c'est le constat empirique d'une condition effective et objective de l'humain, qui elle aussi s'impose aux humains, au point, comme la première au fond, de menacer l'humanisme le plus traditionnel! De même que l'homme rationnel ne se saisit pas comme tel intemporellement mais toujours à travers une histoire croisée des savoirs, de même, il ne se rejoint lui-même qu'à travers le langage qu'il parle et qui est la condition de son accès au monde, c'est-à-dire à toute chose et à quoi que ce soit, y compris lui-même ! Dès lors, FW court délibérément le risque de quitter l'humanisme: car on pourrait bien voir dans l'homme non pas le principe, non pas même un être ou une valeur spécifique mais simplement un effet de langage. Cette condition, cette fois, serait rationnelle, au sens de la logique et de la philosophie analytique qui sous-tendent en effet cette admirable entreprise en dialogue avec les plus exigeantes doctrines contemporaines du langage. Mais serait-elle encore un « humanisme » au sens que nous disions plus haut? En réalité, elle le reste, en raison 
de la troisième dimension que nous verrons dans un instant et d'une autre encore, mais aussi de l'intérieur de son propre développement. Car le langage qui caractérise l'être humain l'inscrit aussi dans l'être à travers sa propre structure, non pas tant comme structure de l'être parlant, mais, dans cette parole, par la place qui y est faite à l'être et même finalement au sujet agissant. Le « je » qui agit selon des raisons : ce n'est pas un postulat de la raison, mais c'est la condition que nous fait le langage dans le monde, selon Francis Wolff. Ainsi, bien loin de nous éloigner de l'humanisme rationnel, l'analyse logique du langage nous y ramène, et même à son aspect normatif. Il rejoint l'aspect normatif de la vie humaine par l'arête ou la face nord de l'analyse la plus exigeante du langage. L'homme a une place dans l'être, par la vertu du langage qui est la condition de l'un comme de l'autre. Condition formelle de l'action, complétant la condition historique du savoir, tel est l'humanisme rationnel original et contemporain de Francis Wolff.

Mais vous attendez bien sûr la troisième dimension. Elle est là, elle vient, elle ne cesse même de se développer dans cette œuvre et lui fait rejoindre comme il se doit le vaste public qu'elle vise depuis le début dans son écriture comme dans son enseignement d'une clarté et d'une rigueur toujours universelles (et souvent, comme nous le reprenons ici nous-mêmes, en trois parties). On ne peut mieux la caractériser que comme anthropologie philosophique. Il s'agit, autrement dit, dans cette troisième facette de l'œuvre, de comprendre la rationalité de l'homme à travers les pratiques de sa vie, qui en semblent le plus souvent bien éloignées! Et pourtant, selon Francis Wolff, c'est bien la raison qui caractérise aussi les pratiques humaines, dans sa relation critique au vivant où elle est prise. C'est elle qui caractérise la relation et la distance avec l'animal, jusque dans la pratique de la «corrida» qui a justifié chez lui une " philosophie » et l'a inscrit dans un débat contemporain que l'on dira « vivant », c'està-dire aussi souvent violent. C'est elle qui caractérise l'art, et en particulier la musique comme « art des sons », c'est-à-dire rationalisation de la sensibilité sonore, dans ce livre au titre lui aussi révélateur comme un défi philosophique : Pourquoi la musique? Mais c'est un projet général qui se dessine ici, et qui consiste à mesurer l'inscription concrète de la raison dans la vie sensible de l'homme qui est en quelque sorte en tension ou en écart constant avec elle. C'est donc bien toujours le même souci, ou la preuve par la convergence des détours : où qu'on se tourne, dans les directions les plus opposées à un rationalisme et à un humanisme simples, du côté de l'histoire, du langage ou de la vie, on retrouve toujours comme un fait contraignant pour le philosophe et pour tout homme 
cette étrange figure de l'homme rationnel. L'immense travail de cet œuvre converge donc bien en ce point.

Mais il nous indique aussi, du même coup la question qu'on peut lui adresser, non pas de l'extérieur et pour le contester, mais de l'intérieur et pour le compléter, cette question qui seule à notre avis peut menacer mais aussi, si on la prend en compte, renforcer encore cet humanisme qui inspire notre admiration et notre gratitude.

Quelle est cette question? Ce n'est pas seulement celle d'un détour, ou d'un écart, ou d'une tension, entre « l'homme » et le rationnel, mais bien du négatif ou d'une négativité qui vient se loger dans l'homme et dans l'humanisme rationnel et qui doit le faire, qui doit être en son principe et en son cœur. Comment d'ailleurs n'y serait-elle pas? On sent, partout dans cette œuvre, et dans de rares textes explicites (comme l'étude des Notions philosophiques sur «le mal») affleurer l'inquiétude devant le négatif qui l'anime de l'intérieur. Mais elle ne vient jamais complètement sur le devant de la scène et on sent lorsqu'elle le fait la « résistance » de l'humanisme rationnel de FW à cette question qui l'habite pourtant de part en part. Il y a bien ce « sensible » que la raison vient normer, humaniser, et esthétiser. Il y a cette action, ce cri : «j'agis », qui intervient dans le «monde ». Et, dans une admirable annexe à la deuxième édition de Dire le monde, la déduction de «l'interdit» anthropologique. Il y a la tragédie à côté de la philosophie dans le moment grec. Il y a surtout, développée de façon admirable à la fin du livre sur l'homme, et désormais de façon explicite, la revendication d'un humanisme universel et cosmopolite contre l'essentialisation de l'homme, ou les autres utopies qui viennent le menacer. Et finalement toute cette pensée affronte le risque de l'irrationnel sous la forme avant tout de l'idéologie et de l'anti-philosophie, du déni des faits et de la raison, de l'histoire, du langage et de la vie.

Mais tout cela n'est pas rapporté à ce qui fait à la fois la spécificité, la normativité et l'humanité de l'humanisme rationnel, à savoir la négativité vitale, morale, politique et historique contre laquelle il se définit et qui en effet l'habite de l'intérieur. Il ne s'agit pas seulement de critiquer une irrationalité extérieure qui peut devenir une menace redoutable, mais aussi d'affronter une négativité intérieure qui définit en effet l'humain et peut se servir en lui de toutes les puissances de la raison. Comment l'humanisme rationnel de FW peut-il mener ce combat? Telle serait notre question ou plus exactement notre demande. 
Elle est amicale, et ce n'est pas secondaire, car c'est là au contraire que tout culmine, dans la doctrine mais aussi dans la pratique de Francis Wolff. Une amitié qui passe par l'institution qui la rend possible, l'École, l'enseignement, le langage même, et qu'il faut défendre, comme il l'a fait et comme il le continue de le faire. Une amitié qui inscrit l'homme rationnel dans l'être. Une page admirable de L'Être, l'homme, le disciple (dont on comprend bien le troisième élément du titre à présent), situe dans cette amitié interhumaine l'animal lui-même et le dieu. Une amitié singulière enfin, personnelle, aurait-on envie de dire, dans nos vies. Il n'est pas nécessaire, pour partager cette doctrine, cette œuvre, cette amitié, d'en partager aussi comme c'est mon cas bien des circonstances, des lieux, des moments et même les initiales qui ont donné lieu dans nos cas à tant de malentendus, qui m'ont permis d'être invité à des conférences sur la corrida tandis que Francis l'était à des colloques sur Bergson, et j'en passe ! Ce n'est pas nécessaire, mais je peux vous dire aussi que le fait de partager ces initiales, cette familiarité, ces malentendus, est inséparable pour moi de celui de partager aussi ces exigences, ces arguments, ces déchirements, d'exprimer mon admiration pour tout cela et ma gratitude pour tout cela et pour tout le reste.

FW (Frédéric Worms) 